\title{
Assessment of early damage of endometrium after artificial abortion by shear wave elastography
}

\author{
Yan Jiao ${ }^{1,2}$, Nianyu Xue ${ }^{3}$, Chunpeng Zou ${ }^{4}$, Xujuan Shui ${ }^{2}$, Hongqing Wang ${ }^{5}$ and Chunhong $\mathrm{Hu}^{1 *}$
}

\begin{abstract}
Objectives: This study aimed to investigate the application of shear wave elastography (SWE) in the early damage detection through assessing the endometrial elasticity after artificial abortion.

Methods: A total of nulliparous women (20-30 years) who received ultrasonography in our hospital were recruited between January 2017 and December 2017. These women were divided into normal control group (NC; $n=65)$, after once artificial abortion group (AOAA; $n=68$ ), after twice artificial abortion group (ATAA; $n=61$ ), and after three times or more (range, 3-6) artificial abortion group (ATTMAA; $n=60$ ). SWE was performed to evaluate the endometrium; Young's modulus of the endometrium was determined and then the endometrial thickness was measured.

Results: Young's modulus of the endometrium increased in the order of NC group, AOAA group, ATAA group, and ATTMAA group, and Young's modulus increased with the increase in the number of artificial abortions $(p<0.05)$. The endometrial thickness in the ATTMAA group was significantly lower than in the NC group, AOAA group, and ATAA group $(p<0.05)$, but there was no marked difference among the NC group, AOAA group, and ATAA group $(p>0.05)$.
\end{abstract}

Conclusions: SWE increases with increasing number of abortions, which may indicate the damage that is done to the endometrium earlier than measurement of the endometrial thickness do.

Keywords: Shear wave elastography, Endometrium, Elasticity, Artificial abortion, Young's modulus

\section{Key points}

- SWE is effective to detect the endometrial elasticity.

- Assessment of endometrial elasticity by SWE may be a better indication in detecting early endometrial damage than measurement of the endometrial thickness.

\section{Introduction}

Artificial abortion is common due to the no pregnancy intention, limited medical resources, and poor reproductive health education [1-3]. In recent years, artificial abortion still has a high prevalence and has become an

\footnotetext{
* Correspondence: huchunhong694@163.com

${ }^{1}$ Department of Radiology, The First Affiliated Hospital of Soochow

University, No. 188 Shizi Street, Suzhou 215006, China

Full list of author information is available at the end of the article
}

important public health problem [4-6]. Artificial abortion may cause mechanical damage to the endometrium, which increases the risk for some complications such as reproductive tract infection, intrauterine adhesions, and secondary infertility. This significantly affects the mental health and quality of life of women [7-9]. The endometrial recovery after artificial abortion is of great importance. In available studies, some clinical characteristics (such as the time of sustained vaginal bleeding, menstrual blood loss, and time to menstrual cycle regularity) and endometrial thickness are employed to evaluate and monitor the endometrial recovery $[10,11]$. However, these factors that indirectly reflect the endometrial recovery and the endometrial thickness may not reflect the endometrial elasticity, which limit their applications in clinical practice. 


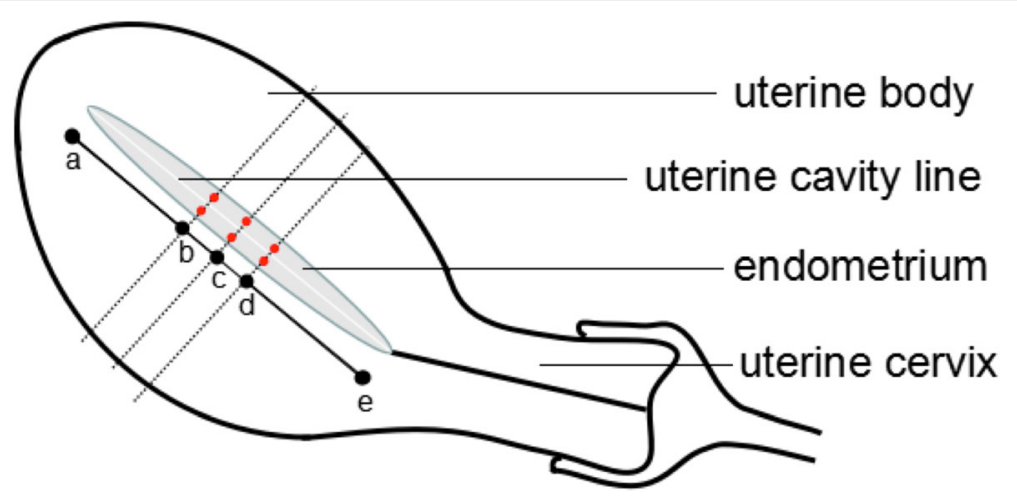

Fig. 1 Sites of endometrium for the measurement of endometrial elasticity by SWE. Notes: Line ae and uterine cavity line are parallel and have the same length; the three dotted lines are vertical to the line ae and endometrial line; point $\mathrm{c}$ is the middle point between point a and point e; point $b$ localizes $0.5 \mathrm{~cm}$ away from the point c (close to a); point d localizes $0.5 \mathrm{~cm}$ away from the point c (close to e). Red dots represent the six sampling sites for SWE.

Shear wave elastography (SWE) is a technique used to evaluate the elasticity of living tissue. It is a real-time, non-invasive, and quantitative technique. In SWE, the probe emits safe acoustic radiation pulses, which can focus on tissues at different depths and continuously induce the tissue particles to vibrate and produce transverse shear wave which is then accurately measured [12]. SWE has been used to evaluate the elasticity of a variety of normal and/or injured tissues [13-15].

Manchanda et al. applied SWE to study the elasticity of normal uterus, and they found no significant difference in the mean endometrial elasticity among women in different menstrual stages or different age groups and no age-related difference in the mean cervical elasticity [16]. Ono et al. revealed that the cervical hardness during pregnancy was negatively related to the gestational age [17]. Zhang et al. found that the myometrial hardness in patients with adenomyosis and leiomyomas was greater than that of the normal myometrium [18]. Currently, little is known about the use of SWE in the detection of elasticity of diseased endometrium. This study aimed to investigate the application of SWE in the assessment of endometrial elasticity after artificial abortion.

\section{Materials and methods Subjects}

A total of 254 nulliparous women (20-30 years) who received gynecological ultrasonography in our hospital were reviewed between January 2017 and December 2017. Their menstrual cycle ranged from 26 to 30 days. According to the number of artificial abortion, these women were divided into normal control group (NC; $n=65$; no abortion; mean age $24.39 \pm 4.26$ years), after once artificial abortion group (AOAA; $n=68$; mean age, $26.01 \pm 3.85$ years), after twice artificial abortion group (ATAA; $n=61$; mean age $25.56 \pm 4.53$ years), and after three times or more (range 3-6) artificial abortion group (ATTMAA; $n=60$; mean age, $24.91 \pm 4.11$ years). Exclusion criteria were as follows: (1) pathology of the uterus, ovary, and fallopian tube; (2) use of drugs that may affect the endometrium (such as estrogen and progesterone)

Table 1 Parameters in different groups

\begin{tabular}{lllllll}
\hline Group & $n$ & Age (years) & Estradiol $(\mathrm{ng} / \mathrm{ml})$ & Progesterone $(\mathrm{pg} / \mathrm{ml})$ & Endometrial thickness $(\mathrm{mm})$ & Young's modulus $(\mathrm{kPa})$ \\
\hline NC & 65 & $24.39 \pm 4.26$ & $8.13 \pm 2.42$ & $295.89 \pm 38.24$ & $11.25 \pm 2.81$ & $10.17 \pm 3.81$ \\
AOAA & 68 & $26.01 \pm 3.85$ & $8.35 \pm 2.31$ & $306.16 \pm 36.37$ & $12.03 \pm 2.59$ & $17.01 \pm 4.63^{*}$ \\
ATAA & 61 & $25.56 \pm 4.53$ & $7.80 \pm 2.55$ & $298.35 \pm 37.66$ & $11.57 \pm 2.75$ & $23.35 \pm 4.92^{*}{ }^{*}$ \\
ATTMAA & 60 & $24.91 \pm 4.11$ & $7.98 \pm 2.29$ & $293.13 \pm 40.08$ & $8.25 \pm 2.06^{*} \# \Delta$ & $32.17 \pm 5.38^{* \# \Delta}$ \\
$P_{\text {AOAA vs NC }}$ & & 0.07 & 0.59 & 0.11 & 0.10 & 0.00 \\
$P_{\text {ATAA vs NC }}$ & 0.14 & 0.46 & 0.72 & 0.52 & 0.00 \\
$P_{\text {ATTMAA vs NC }}$ & 0.49 & 0.72 & 0.69 & 0.00 & 0.00 \\
$P_{\text {ATAA vs AOAA }}$ & 0.55 & 0.20 & 0.23 & 0.33 & 0.00 \\
$P_{\text {ATTMAA vs AOAA }}$ & 0.12 & 0.37 & 0.06 & 0.00 & 0.00 \\
$P_{\text {ATTMAA vs ATAA }}$ & 0.41 & 0.68 & 0.46 & 0.00 & 0.00 \\
\hline
\end{tabular}

${ }^{*} p<0.05$ vs NC group; ${ }^{\#} p<0.05$ vs AOAA group; ${ }^{\Delta} p<0.05$ vs ATAA group 

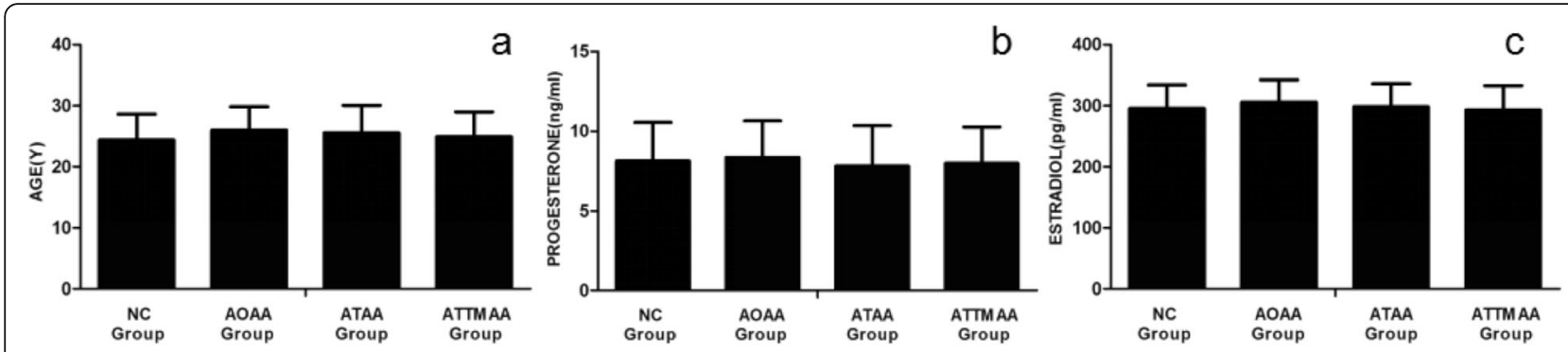

Fig. 2 Age, progesterone, and estradiol in the NC group, AOAA group, ATAA group, and ATTMAA group. a age; b progesterone; c estradiol

within 1 month before ultrasonography; (3) presence of uterine effusion, intrauterine adhesions, and intrauterine residual or normal menstrual cycle within 3 months after the last artificial abortion. The study was conducted in accordance with the Committee for Human Research at our institution and followed all regulations. Informed consent was obtained before the study.

\section{Instruments and methods}

Supersonic Imagine AixPlorer ultrasonic instrument with SWE (Shear Wave ${ }^{\mathrm{TM}}$ ) was used for the detection of endometrial elasticity. The SE12-3 probe (frequency 3$12 \mathrm{MHz}$ ) applicable for SWE was used.

For subjects, SWE of the endometrium was performed by the same sonographer. Meanwhile the examination was performed 3 months after the last abortion. The endometrial elasticity was evaluated in the ovulation stage (days 14-16 of menstrual cycle; maximal diameter of follicles, $18-22 \mathrm{~mm}$ ). In brief, women lied in a supine position, and routine abdominal ultrasonography was performed to explore the pelvic organs. Then, transvaginal two-dimensional ultrasonography was done to observe the shape, outline, and internal structures of the uterus after emptying the bladder. Thereafter, transvaginal ultrasonography was done at multiple views to evaluate the symmetry of the uterus, possible presence of uterine effusion and other lesions, and assess the endometrial echoes and thickness as well as the borderline between the endometrium and myometrium. The endometrial thickness was measured at the maximal longitudinal section and recorded. Measurement was done at the middle point between the fundus of uterus and cervix. Then, SWE of the endometrium was performed with the SWE mode. The red, green, and blue colors represent high, intermediate, and low Young's modulus, respectively. When the images became stable, the images were frozen, and quantification was done with the Q-BOX system. Young's modulus of the anterior and posterior endometrium was measured. The diameter of region of interest (ROI) was $2 \mathrm{~mm}$, and the distance between ROI and probe was $2-4 \mathrm{~cm}$. The ROI was set at three sites of the anterior endometrium and three sites of the posterior endometrium (middle point between the fundus of uterus and cervix, $0.5 \mathrm{~cm}$ away from the

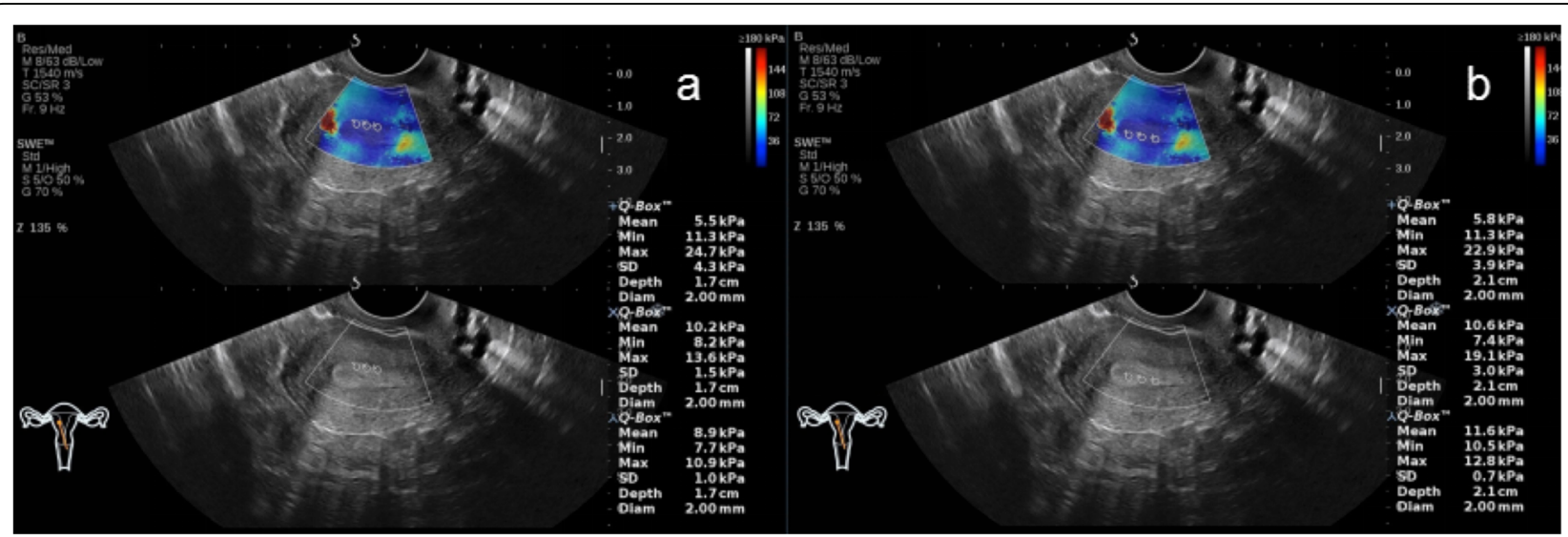

Fig. 3 Representative images of a woman in the NC group. a Young's modulus at the three sites of the anterior endometrial wall was 5.5, 10.2, and $8.9 \mathrm{kPa}$, respectively; $\mathbf{b}$ Young's modulus at the three sites of the posterior endometrial wall was 5.8, 10.6, and 11.6 kPa, respectively. The mean Young's modulus of the six sampling sites was $8.8 \mathrm{kPa}$ 


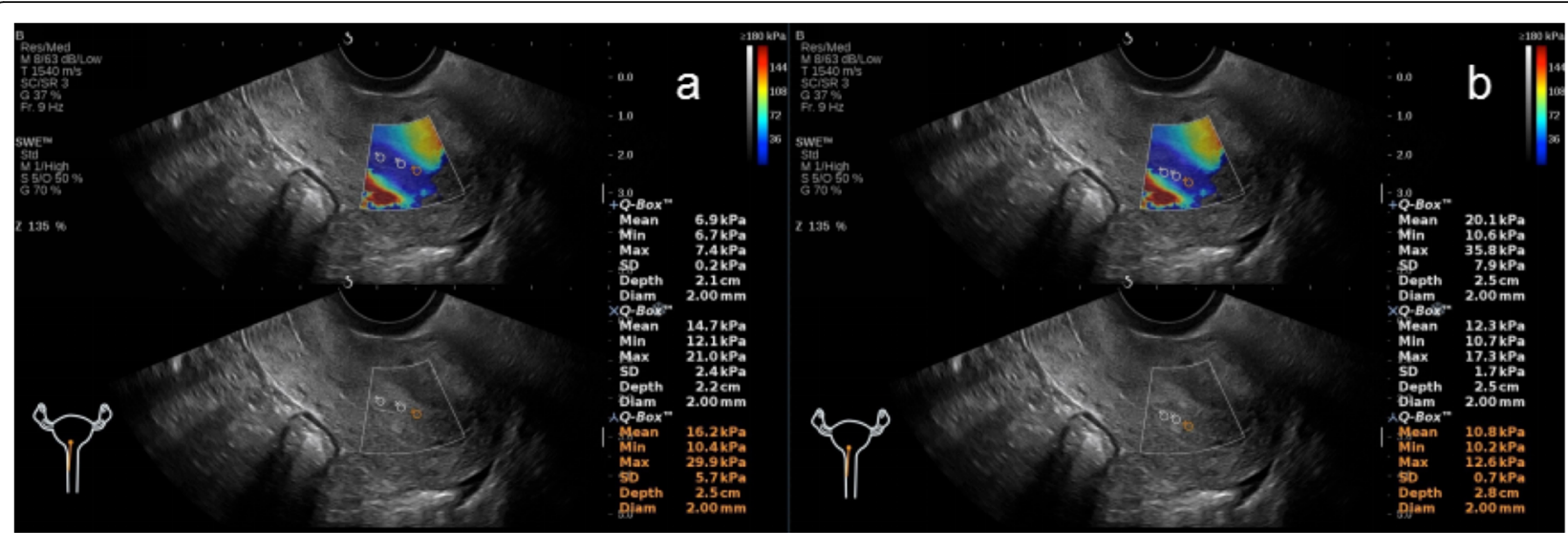

Fig. 4 Representative images of a woman in the AOAA group. a Young's modulus at the three sites of the anterior endometrial wall was 6.9, 14.7, and $16.2 \mathrm{kPa}$, respectively; $\mathbf{b}$ Young's modulus at the three sites of the posterior endometrial wall was 20.1, 12.3, and $10.8 \mathrm{kPa}$, respectively. The mean Young's modulus of the six sampling sites was $13.5 \mathrm{kPa}$

middle point [close to the fundus of uterus] and 0.5 $\mathrm{cm}$ away from the middle point [close to the cervix]) (Fig. 1). All these ROIs localized at the middle points between endometrium-myometrium boundary line and uterine cavity line. The mean, maximal, and minimal Young's moduli were automatically calculated by the Q-BOX system. The mean Young's modulus of six sites was determined. At the same time, blood was collected on the day of ultrasonography, and the serum levels of estradiol and progesterone were detected and recorded.

\section{Statistical analysis}

Data were expressed as mean \pm standard deviation (SD). Statistical analyses were performed using the statistical package for social software version 22 (SPSS Inc., Chicago, IL, USA). Comparisons of quantitative data were done using one-way analysis of variance (ANOVA) followed by post hoc least significant difference (LSD) test. A value of $p<0.05$ was considered statistically significant.

\section{Results}

There were no marked differences in age and serum levels of progesterone and estradiol among the NC group, AOAA group, ATAA group, and ATTMAA group $(P>0.05)$ (Table 1; Fig. 2).

In the NC group, AOAA group, ATAA group, and ATTMAA group, the endometrial thickness was $11.25 \pm$ $2.81 \mathrm{~mm}, 12.03 \pm 2.59 \mathrm{~mm}, 11.57 \pm 2.75 \mathrm{~mm}$, and $8.25 \pm$ $2.06 \mathrm{~mm}$, respectively. The endometrial thickness in the ATTMAA group was significantly lower than in the NC group, AOAA group, and ATAA group $(p<0.05)$, but there was no marked difference among the $\mathrm{NC}$ group, AOAA group, and ATAA group $(p>0.05)$. In the NC group, AOAA group, ATAA group, and ATTMAA

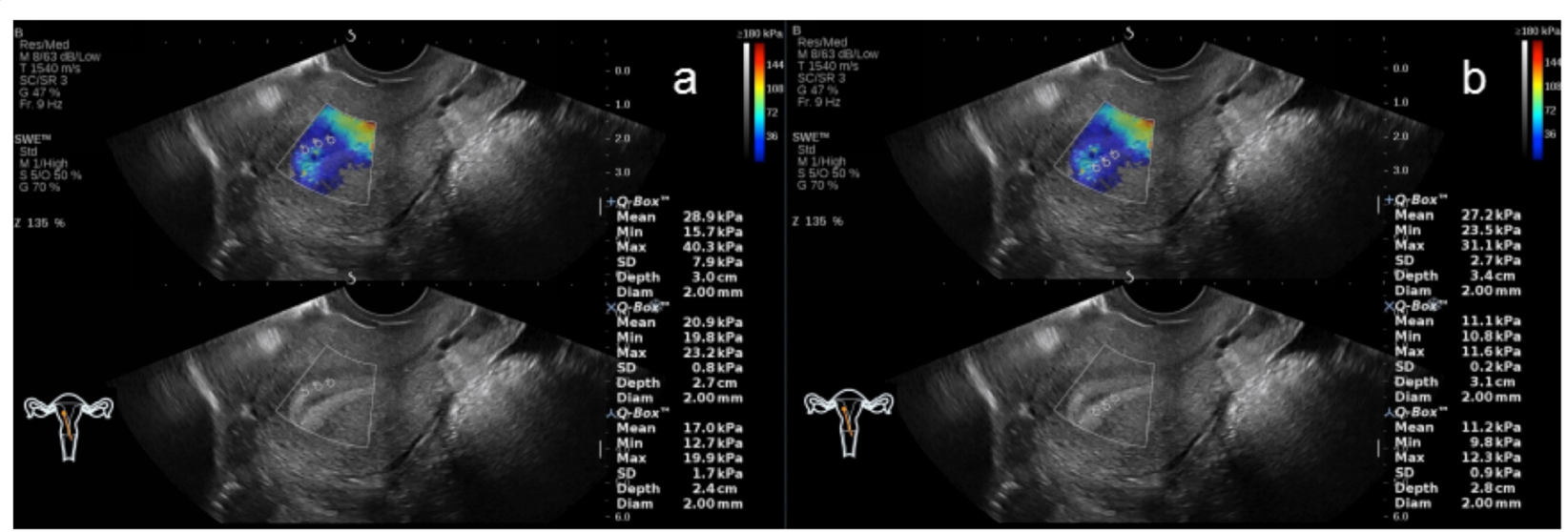

Fig. 5 Representative images of a woman in the ATAA group. a Young's modulus at the three sites of the anterior endometrial wall was 28.9, 20.9, and $17.0 \mathrm{kPa}$, respectively; b Young's modulus at the three sites of the posterior endometrial wall was 27.2, 11.1, and 11.2 kPa, respectively. The mean Young's modulus of the six sampling sites was $19.4 \mathrm{kPa}$ 


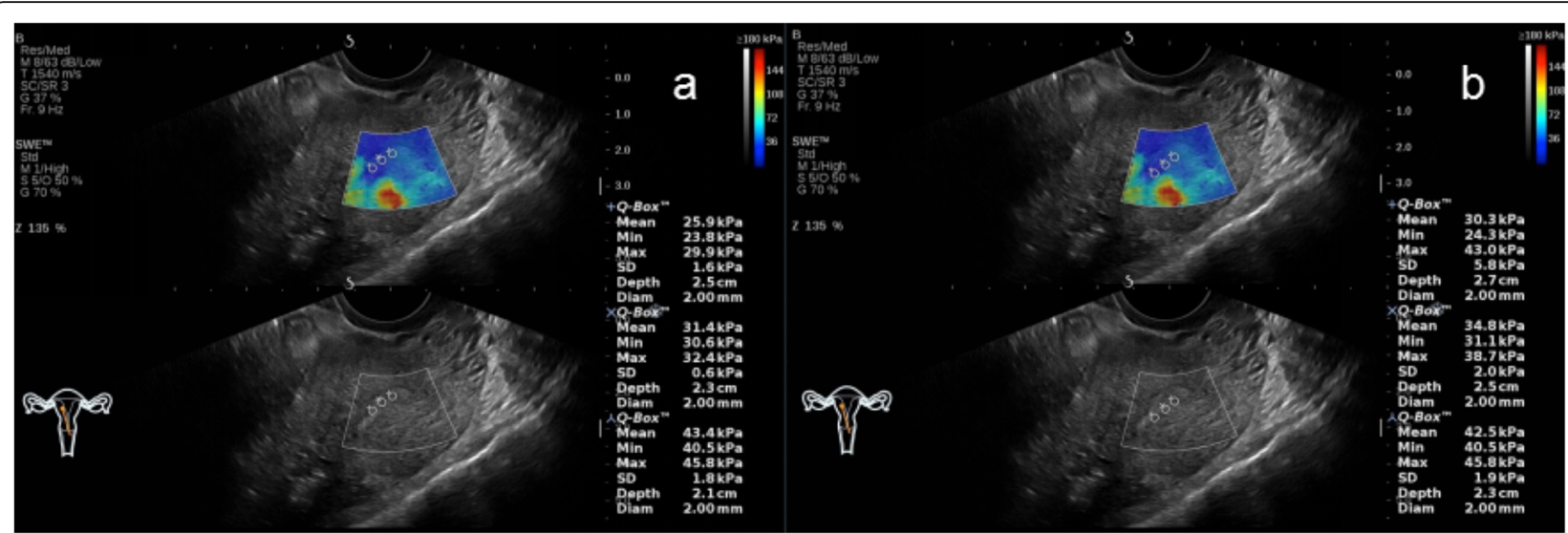

Fig. 6 Representative images of a woman in the ATTMAA group. a Young's modulus at the three sites of the anterior endometrial wall was 25.9, 31.4 , and $43.4 \mathrm{kPa}$, respectively; b Young's modulus at the three sites of the posterior endometrial wall was $30.3,34.8$, and $42.5 \mathrm{kPa}$, respectively. The mean Young's modulus of the six sampling sites was $34.7 \mathrm{kPa}$

group, Young's modulus was $10.17 \pm 3.81 \mathrm{kPa}, 17.01 \pm$ $4.63 \mathrm{kPa}, 23.35 \pm 4.92 \mathrm{kPa}$, and $32.17 \pm 5.38 \mathrm{kPa}$, respectively. Young's modulus was significantly different between any two groups $(p<0.05)$ (Figs. 3, 4, 5 and 6). Young's modulus increased with the increase in the number of artificial abortion (Table 1; Fig. 7).

\section{Discussion}

In the present study, the endometrial thickness and elasticity were assessed in post-abortion patients and normal subjects. Results showed that endometrial thickness in the ATTMAA group was significantly lower than in the NC group, AOAA group, and ATAA group, but there was no marked difference among the AOAA group, ATAA group, and NC group. In addition, Young's modulus increased significantly in the order of NC group, AOAA group, ATAA group, and ATTMAA group. These findings indicate that the endometrial elasticity reduces after repeated artificial abortion in the ATTMAA group; although the endometrial thickness in the AOAA group and ATAA group remains unchanged, the physical characteristics of the endometrium change significantly (elasticity reduces and stiffness increases), which was more evident in the TAA group than in the AOAA group.

Some investigators found the mechanical damage to the endometrium could reduce the endometrial glandular epithelium and increase the collagen fibers in animal model of artificial abortion [19], leading to the increased proportion of collagen fibers in the endometrium. There is evidence showing that the increased collagen fibers in the organ may significantly compromise the elasticity of this organ and increases its stiffness [20,21]. Thus, on the basis of our findings, we speculate that the mechanical damage to the endometrium after artificial abortion may cause hyperplasia of collagen fibers in the endometrium and thereafter increase their amount, which leads to the increase in the endometrial stiffness [22-24]. Moreover, the endometrial stiffness increases with the increase in the number of artificial abortion. The
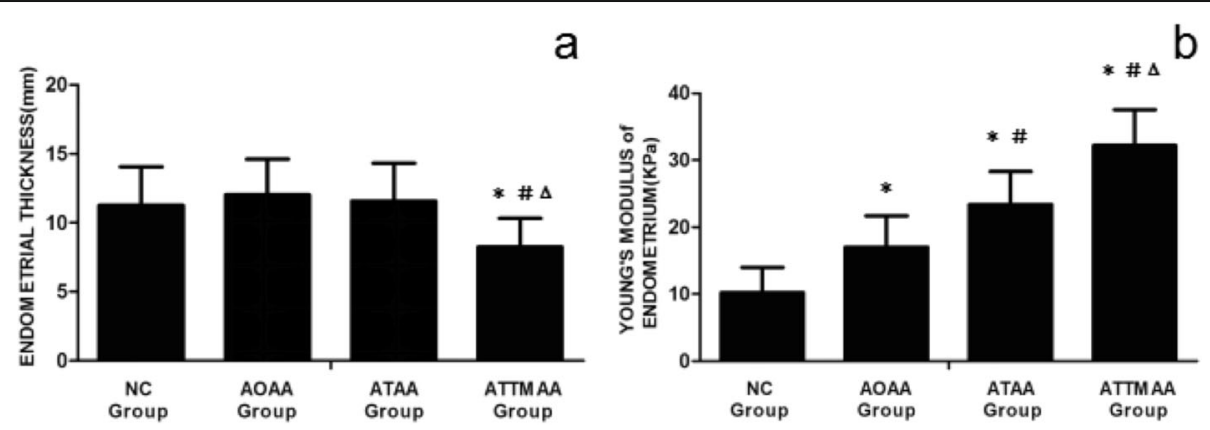

Fig. 7 Young's modulus endometrium and endometrial thickness in the NC group, AOAA group, ATAA group, and ATTMAA group. a Endometrial thickness in the NC group, AOAA group, ATAA group, and ATTMAA group. $\mathbf{b}$ Young's modulus of the endometrium in the NC group, AOAA group, ATAA group, and ATTMAA group. ${ }^{*} p<0.05$ vs NC group. ${ }^{*} p<0.05$ vs AOAA group. ${ }^{{ }} p<0.05$ vs ATAA group 
pathological changes in the endometrium are consistent with the change in the endometrial stiffness. Recent studies have shown that SWE can be used to evaluate the elasticity of normal myometrium, intima, and cervix and that SWE is a promising tool for the uterine assessment and helpful for the diagnosis of various uterine diseases [16]. Available findings together with our results indicate that SWE may be used to display the early damage to the endometrium when it is invisible on conventional ultrasonography and 2D measurements. Taking this research as an opportunity, we can carry out more systematic studies on the application of SWE in the assessment of endometrium after artificial abortion. This is a direction of our future investigations.

There were limitations in this study. The uterus is three-dimensional, and current techniques cannot sample the endometrium at different sites simultaneously. Thus, the middle points of the anterior and posterior walls of the uterus were selected. We believe that the development of medical technology will improve the measurement of endometrial elasticity. However, our findings provide valuable information for the clinical assessment of endometrial damage after artificial abortion, which is helpful for the diagnosis and management of endometrial complications after abortion.

Taken together, SWE is effective to detect the endometrial elasticity after artificial abortion, which seems to indicate the assessment of physical characteristics and pathological changes of the endometrium. The changes in the endometrium after artificial abortion may occur preceding the clinical manifestations and the alteration of endometrial thickness. Thus, the endometrial elasticity determined by SWE may provide important information for the assessment of endometrial recovery.

\section{Abbreviations}

AOAA: After once artificial abortion; ATAA: After twice artificial abortion; ATTMAA: After three times or more artificial abortion; ROI: Region of interest; SD: Standard deviation; SWE: Shear wave elastography

\section{Authors' contributions}

YJ and $\mathrm{CH}$ designed this study. YJ, NX, CZ, XS, HW, and CH collected and interpreted the patients' data. YJ analyzed the data. YJ was the major contributor to the writing of the manuscript. All authors read and approved the final manuscript.

\section{Funding}

This study was supported by the Basic and Public Studies of Zhejiang Province (No. LGF18H180003) and the Health and Family Planning Commission of Zhejiang Province, China (Nos. 2018ZD043 and 2019KY665).

\section{Availability of data and materials}

The datasets used and/or analyzed in the present study are available from the corresponding author on reasonable request.

\section{Ethics approval and consent participate}

The study was conducted in accordance with the Committee for Human Research at our institution and followed all regulations. Informed consent was obtained before study.

\section{Consent for publication}

A consent to publication has been signed by each participant.

\section{Competing interests}

The authors declare that they have no competing interests.

\section{Author details}

${ }^{1}$ Department of Radiology, The First Affiliated Hospital of Soochow University, No. 188 Shizi Street, Suzhou 215006, China. ${ }^{2}$ Obstetrics and Gynecology Ultrasonic Department, Wenzhou Peoples' Hospital, Wenzhou 325000, China. ${ }^{3}$ Department of Diagnostic Ultrasonography, Ningbo First Hospital, Ningbo 315010, China. ${ }^{4}$ Department of Diagnostic Ultrasonography, The Second Affiliated Hospital and Yuying Children's Hospital of Wenzhou Medical University, Wenzhou 325027, China. ${ }^{5}$ Department of Radiology, The First Affiliated Hospital of Wenzhou Medical University, Wenzhou 325000, China.

Received: 9 September 2019 Accepted: 30 January 2020

Published online: 04 March 2020

\section{References}

1. Faundes A, Shah $\mathbb{H}$ (2015) Evidence supporting broader access to safe legal abortion. Int J Gynaecol Obstet 131(Suppl 1):S56-S59

2. Maina BW, Mutua MM, Sidze EM (2015) Factors associated with repeat induced abortion in Kenya. BMC Public Health 15:1048

3. Pereira J, Pires R, Canavarro MC (2017) Psychosocial adjustment after induced abortion and its explanatory factors among adolescent and adult women. J Reprod Infant Psychol 35:119-136

4. Erdman JN (2012) Harm reduction, human rights, and access to information on safer abortion. Int J Gynaecol Obstet 118:83-86

5. Labandera A, Gorgoroso M, Briozzo L (2016) Implementation of the risk and harm reduction strategy against unsafe abortion in Uruguay: from a university hospital to the entire country. Int J Gynaecol Obstet 134:S7-s11

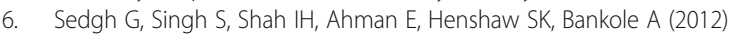
Induced abortion: incidence and trends worldwide from 1995 to 2008. Lancet 379:625-632

7. Gerdts C, Hudaya I (2016) Quality of care in a safe-abortion hotline in Indonesia: beyond harm reduction. Am J Public Health 106:2071-2075

8. Russo JA, Achilles S, DePineres T, Gil L (2013) Controversies in family planning: postabortal pelvic inflammatory disease. Contraception 87:497-503

9. Xiao S, Wan Y, Xue M et al (2014) Etiology, treatment, and reproductive prognosis of women with moderate-to-severe intrauterine adhesions. Int J Gynaecol Obstet 125:121-124

10. Madeiro AP, Rufino AC (2017) Maltreatment and discrimination in induced abortion care: perception of women in Teresina, State of Piaui, Brazil. Cien Saude Colet 22:2771-2780

11. Rottenstreich A, Amsalem H, Kleinstern G, Kalish Y (2017) Outcomes of threatened abortions after anticoagulation treatment to prevent recurrent pregnancy loss. Reprod Biomed Online 35:461-467

12. Taljanovic MS, Gimber LH, Becker GW et al (2017) Shear-wave elastography: basic physics and musculoskeletal applications. Radiographics 37:855-870

13. Barr RG (2018) Shear wave liver elastography. Abdom Radiol (NY) 43:800-807

14. Hu X, Liu Y, Qian L (2017) Diagnostic potential of real-time elastography (RTE) and shear wave elastography (SWE) to differentiate benign and malignant thyroid nodules: a systematic review and meta-analysis. Medicine (Baltimore) 96:e8282

15. Liu H, Wan J, Xu G et al (2019) Conventional US and 2-D shear wave elastography of virtual touch tissue imaging quantification: correlation with immunohistochemical subtypes of breast cancer. Ultrasound Med Biol 45:2612-2622

16. Manchanda S, Vora Z, Sharma R et al (2019) Quantitative sonoelastographic assessment of the normal uterus using shear wave elastography: an initial experience. J Ultrasound Med 38:3183-3189

17. Ono T, Katsura D, Yamada K et al (2017) Use of ultrasound shear-wave elastography to evaluate change in cervical stiffness during pregnancy. J Obstet Gynaecol Res 43:1405-1410

18. Zhang M, Wasnik AP, Masch WR et al (2019) Transvaginal ultrasound shear wave elastography for the evaluation of benign uterine pathologies: a prospective pilot study. J Ultrasound Med 38:149-155 
19. Huberlant S, Fernandez H, Vieille P et al (2015) Application of a hyaluronic acid gel after intrauterine surgery may improve spontaneous fertility: a randomized controlled trial in New Zealand White rabbits. PLoS One 10: e0125610

20. Lunde IG, Herum KM, Carlson CC, Christensen G (2016) Syndecans in heart fibrosis. Cell Tissue Res 365:539-552

21. Voorhees AP, DeLeon-Pennell KY, Ma Y et al (2015) Building a better infarct: modulation of collagen cross-linking to increase infarct stiffness and reduce left ventricular dilation post-myocardial infarction. J Mol Cell Cardiol 85:229-239

22. Brabrand A, Kariuki II, Engstrom MJ et al (2015) Alterations in collagen fibre patterns in breast cancer. A premise for tumour invasiveness? APMIS 123:1-8

23. Liu H, Zhao LX, Xu G et al (2015) Diagnostic value of virtual touch tissue imaging quantification for benign and malignant breast lesions with different sizes. Int J Clin Exp Med 8:13118-13126

24. Wang ZL, Li JL, Li M, Huang Y, Wan WB, Tang J (2013) Study of quantitative elastography with supersonic shear imaging in the diagnosis of breast tumours. Radiol Med 118:583-590

\section{Publisher's Note}

Springer Nature remains neutral with regard to jurisdictional claims in published maps and institutional affiliations.

\section{Submit your manuscript to a SpringerOpen ${ }^{\circ}$ journal and benefit from:}

- Convenient online submission

- Rigorous peer review

- Open access: articles freely available online

- High visibility within the field

Retaining the copyright to your article

Submit your next manuscript at $\boldsymbol{\wedge}$ springeropen.com 